\title{
Children with cerebral palsy do not achieve healthy physical activity levels
}

\author{
L. U. Bratteby Tollerz, A. H. Forslund, R. M. Olsson, Helene Lidström and U. Holmback
}

\section{Linköping University Post Print}

\section{Tweet}

N.B.: When citing this work, cite the original article.

Original Publication:

L. U. Bratteby Tollerz, A. H. Forslund, R. M. Olsson, Helene Lidström and U. Holmback, Children with cerebral palsy do not achieve healthy physical activity levels, 2015, Acta Paediatrica, (104), 11, 1125-1129.

http://dx.doi.org/10.1111/apa.13141

Copyright: Wiley: 12 months

http://eu.wiley.com/WileyCDA/

Postprint available at: Linköping University Electronic Press

http://urn.kb.se/resolve?urn=urn:nbn:se:liu:diva-122783 


\section{Children with cerebral palsy do not achieve healthy physical activity levels}

Short title: Physical activity and cerebral palsy

Authors

Bratteby Tollerz $\mathrm{LU}^{1,2^{*}}$, Forslund $\mathrm{AH}^{2}$, Olsson $\mathrm{RM}^{1}$, Lidström $\mathrm{H}^{3}$, Holmbäck $\mathrm{U}^{1}$ Institutional affiliations

1. Department of Public Health and Caring Sciences and Departments of Women`s and Children`s Health, (S-75185) Uppsala, Sweden.

2. Department of Women`s and Children`s Health and Public Health and Caring Sciences, Uppsala University, (S-75185) Uppsala, Sweden.

3. Department of Public Health and Caring Sciences, Uppsala University, (S75185) Uppsala, Sweden.

4. Department of Women`s and Children`s Health, Uppsala, Sweden.

Department of Social and Welfare Studies, Linköping University, Norrköping, Sweden

* Corresponding author

Linda U Bratteby Tollerz, MSc, PT

Akademiska Sjukhuset

Kvinno- och barndivisionen, S-751 85 Uppsala, Sweden

E-mail: linda.bratteby.tollerz@akademiska.se

Mobile phone 0046703959001 


\section{ABSTRACT}

Aim: This study compared daily activity energy expenditure (AEE) in children with cerebral palsy with a control group and investigated whether the children achieved healthy levels of physical activity.

Methods: We enrolled eight children with bilateral cerebral palsy, from eight to 10 years of age, and a group of controls matched for age and gender. For three days physical activity was simultaneously measured by accelerometers and self-reports using a diary. The daily AEE results were compared between groups and methods. The number of children that achieved healthy physical activity levels in each group was explored.

Results: Children with cerebral palsy had significantly lower daily AEE, as measured by accelerometers, than the controls and they did not achieve the healthy moderate to heavy physical activity level defined in the Nordic Nutrition Recommendations. Self-reports using the diaries resulted in an overestimation of physical activity compared with the ankle accelerometer measurements in both groups.

Conclusion: Our investigation of physical activity in children with cerebral palsy and controls using accelerometers and a diary, found low levels of daily AEE and physical activity and these results were most prominent in the group with cerebral palsy. The diaries overestimated physical activity in both groups.

Keywords: Accelerometer, Activity energy expenditure, Cerebral palsy, Children, Physical activity level

\section{Key notes}

- This study compared daily activity energy expenditure in children with cerebral palsy with a control group and investigated whether the children achieved healthy levels of physical activity. 
- Accelerometer measurements show lower daily activity energy expenditure and physical activity levels in eight children with cerebral palsy than eight matched controls.

- Self-reports using a diary overestimated physical activity in both groups when they were compared with accelerometer readings.

\section{INTRODUCTION}

Physical inactivity is a global problem in modern society and it has been shown to lead to serious health problems (1). It is associated with illness in children and it is involved in the development of type 2 diabetes and cardiovascular disease (2). In contrast, healthy levels of physical activity promote good physical health and are important for normal growth and the development of motor skills (3). According to the Nordic Nutrition Recommendations (NNR), children should accumulate 60 minutes daily of physical activity at a moderate to heavy intensity level (4). Moreover, research has shown that physical activity improves children's self-esteem, social interactions and weight control and reduces stress (5).

Ideally, daily energy intake should correspond to total energy expenditure (TEE), which is the sum of an individual's basal metabolic rate (BMR), their daily activity energy expenditure (AEE) and their diet-induced thermogenesis (DIT). Of these three components, DIT accounts for about $10 \%$ of the TEE (4). The BMR is also relatively stable and it can be estimated based on an individual's age and weight, for example using the Schofield equation. These estimations have been found to be in good agreement with measurements by indirect calorimeter and the Schofield equation is commonly used in children (6). Therefore, the daily AEE directly affects TEE and the more we know about an individual's daily $A E E$, the better our recommendations to balance their energy expenditure and energy intake will be.

Several studies have shown that inactivity in children with cerebral palsy is substantial $(7,8)$, but little is known about their daily AEE and it is essential to investigate whether they reach a sufficient level of physical activity and balanced energy intake. Cerebral 
palsy is defined as a group of permanent neurological motor disorders, limiting physical activity. These non-progressive disturbances occur in the developing fetal or infant brain (9). Motor function is affected to different degrees, depending on the extent of the neurological impairment (10) and individual functional level can be classified using the Gross Motor Function Classification System (11). Motor impairment in combination with decreased physical fitness due to inactivity will affect these individuals' ability to engage in daily activities and this may lead to various secondary conditions, such as fatigue and pain (12). These problems also seem to increase with age, especially for girls who are less active than boys regardless of age or ethnicity (13). Children with cerebral palsy often suffer from feeding difficulties and medical problems during childhood. This can have an impact on the parental burden and may interfere with time spent on physical activity (9). Moreover, a common problem for children with cerebral palsy is the lack of available adjusted physical activities at a sufficiently moderate to heavy level (14).

In children with cerebral palsy, energy expenditure is increased during physical activity in comparison to controls $(15,16)$, which could lead to the assumption that they also achieve high daily AEE and healthy levels of physical activity.

Physical activity can be quantified by self-reports such as diaries and questionnaires, which are indirect methods, or direct methods such as heart rate monitoring, pedometers and accelerometers (17). Health professionals' recommendations for physical activity to promote health in children with cerebral palsy are usually based on indirect methods, such as information given by the child or caregivers and not on any objective investigation of the child's daily AEE (17). It is unclear how well one can assess whether the individual has achieved a satisfactory physical activity level based on self-reports.

Triaxial accelerometers have been shown to provide the best assessment of activity and oxygen consumption of the three direct methods listed above (18) and placing the accelerometer on the lower extremities has been shown to effectively capture daily physical activity (19). 
To promote health in children with cerebral palsy there is a need to explore suitable methods for valid and reliable measurement of physical activity. The aims of this study were to compare daily AEE between children with cerebral palsy and a control group by a direct and indirect method and in addition to investigate whether the children achieved healthy physical activity levels according to the NNR (4).

\section{METHODS}

The approach was a descriptive and comparative study in children with cerebral palsy and a matched group of healthy controls. The study was conducted at the Clinical Nutrition and Metabolism Laboratory in Uppsala, Sweden. Data were collected between 2006 and 2010 after approval from the Ethics Board of Uppsala University (Log No. 2006/311).

\section{Participants}

We enrolled 16 children, eight with cerebral palsy and eight controls matched for age and gender to the study. Children with cerebral palsy were recruited by the first author (LBT) as a convenience sample in conjunction with treatment sessions at a local rehabilitation centre. Inclusion criteria were that the children and their families had given informed consent, the children were able to walk for 10 minutes with or without a walking aid, they could understand given instructions, had a body mass index (BMI) within $+/-2$ standard deviation (SD) of mean BMI adjusted for age according to Karlberg (20) and were in good health on the day of examination. Seven children in the study group were diagnosed with bilateral cerebral palsy at level II or III according to the Gross Motor Function Classification System (11) and one presented with the same motor impairment after traumatic brain injury. The control group was recruited from the families of colleagues and friends. The children were seven to 12 years of age and there were five boys and three girls in each group. All families were approached in person and given an information letter. If a signed consent form was sent back an appointment was arranged. 


\section{Procedures}

The children arrived at the Clinical Nutrition and Metabolism Laboratory between 8am and 9am on a weekday between Monday and Thursday. They had all been fasting since midnight. First, they were asked about their health status and their body weight and height were registered. Children with cerebral palsy were measured supine and the others were standing. The children were given the option to watch a movie while their BMR was measured by indirect calorimeter (Vmax Encore, SensorMedics, CareFusion Corp., San Diego, California, USA). Instructions were given on how to do the self-reporting in the diary and the child and family practiced self-reporting by retrospectively entering all activity since the child got out of bed that morning. The activities were categorised into nine levels, ranging from one for sleep and rest to nine for maximal physical activities. The diary was then continued for the rest of the day and for two more consecutive days with support from adults at home or in school. The diary had previously been validated by the doubly labelled water method in adolescents and provided a close estimation of the TEE (21).

Finally, the accelerometers were applied to the wrist of the writing hand and the right ankle for the controls and on both wrists and ankles for the children with cerebral palsy to enable comparison between the two sides. Data were collected by measuring counts at 15second intervals for three days.

Individual daily energy expenditure was calculated as the measured BMR (BMRm) added to the daily AEE measured by the ankle accelerometer or the two accelerometers in the case of the study group. These results were compared with the daily energy expenditure values based on the estimated BMR, based on the Schofield equation, and estimated daily AEE, based on the diary. The light and heavy activity level were set at $30 \mathrm{~kJ} / \mathrm{kg} / 24 \mathrm{~h}$ below or above the moderate level according to the NNR (4).

The sensitivity of the Actical accelerometer (Mini Mitter Co., Bend, Oregon, USA) was set to collect movements in the $0.5-$ to $3-\mathrm{Hz}$ range. Cut-off points for registered activity counts, depending on placement, height, length of bone, and step frequency, have been published for children from eight to 17 years of age (22). 


\section{Statistical analysis}

Due to the small sample size, the Mann-Whitney $U$ test without continuity correction was used to test for differences between groups. The two comparisons of daily AEE between the children with cerebral palsy and controls, measured by the ankle accelerometers and diaries, were considered to be the primary analyses and adjustment for multiple testing was carried out according to Holm (23). Other comparisons were considered to be exploratory and no adjustment for multiple testing was carried out. Two children with cerebral palsy were excluded from the analysis of the accelerometer data because they refused to wear them. A sensitivity analysis due to these missing values was carried out by imputing data from matched controls. One child with cerebral palsy wore the wrist accelerometers for two days instead of three and these data were included, but no adjustments were made in the analysis. Results from the different methods for assessment of daily AEE were compared using the Wilcoxon matched pairs test. Significance was set at the 5\% level. Statistica 11 (StatSoft Inc., Tulsa, Oklahoma, USA) was used for the statistical analysis.

\section{RESULTS}

There was no missing data in the diaries, which enabled us to compare all eight children in each group. Two children in each group had removed the accelerometers during swimming. The AEE for this activity was calculated from the diary and used to complete the accelerometer data. There was no difference between the measured and calculated BMR within and between the groups (Table 1).

There was a lower daily AEE in the group with cerebral palsy compared to the control group, irrespective of method (Table 2). When we compared the daily AEE measured by the ankle accelerometers, the study group's results were on average 2,092 kJ/24h (498 $\mathrm{kcal} / 24 \mathrm{~h}$ ) lower than the controls. Similarly, the study group had lower daily AEE values based on the diary and the average difference was $1541 \mathrm{~kJ} / 24 \mathrm{~h}$ (367 kcal/24h) between groups. 
None of the children with cerebral palsy reached the moderate or heavy level of physical activity, as measured by the accelerometers, and only five children in the control group did. On the other hand, four children in the study group and seven in the control group reported reaching these recommended levels in their diaries. The individual results are shown in the Appendix S1.

\section{Comparison of methods}

The average daily AEE was $1,205 \mathrm{~kJ} / 24 \mathrm{~h}(287 \mathrm{kcal} / 24 \mathrm{~h})(\mathrm{p}=0.001)$ higher in the diaries than the measurements obtained by the ankle accelerometers. In contrast, no differences between daily AEE based on the diaries and the wrist accelerometers were found. When we compared the discrepancy between the measured and reported daily $A E E$, there was no difference between the groups and the respective findings were: $1,252 \mathrm{~kJ} / 24 \mathrm{~h}(298 \mathrm{kcal} / 24 \mathrm{~h})$ $(p=0.028)$ and $1,172 \mathrm{~kJ} / 24 \mathrm{~h}(279 \mathrm{kcal} / 24 \mathrm{~h})(p=0.017)$.

\section{DISCUSSION}

Children with cerebral palsy had significantly lower daily AEE levels, based on accelerometer measurements and diaries, than their age and gender-matched controls, indicating lower levels of physical activity. Self-reports in the diaries overestimated daily AEE when they were compared with the measurements from the ankle accelerometers. Neither of the children with cerebral palsy or the children in the control group achieved healthy physical activity levels.

The results are in line with other studies that revealed low levels of physical activity in children with cerebral palsy $(7,8)$.

Ankle accelerometer measurements showed a lower daily AEE than the diary. Assuming that the accelerometers placed on the ankles provided a more objective method and measured low-intensity physical activity accurately (19), the diary appeared to overestimate daily AEE. Overestimated self-reports of physical activity have previously been reported $(18,24)$. The observation that participants in both groups overestimated both the grade of intensity and the duration of the activity could raise questions about the general 
fitness of the control group. Notably, daily AEE measurements showed that the control group did not achieve the recommended level of physical activity during the study either. Other possible explanations for the difference between the diaries and the accelerometers could be that participation in the study heightened awareness of physical activity, but also that the devices could have affected the study subjects' perceptions as well as changed their habitual activity patterns. The subjects reported that they were more encumbered by the diaries than the accelerometers. Furthermore, the accelerometers were not accepted by two of the children with cerebral palsy. A more thorough introduction or wearing fewer than four accelerometers could have had a less severe effect on the group with cerebral palsy. It is important to be aware of the weaknesses of indirect methods in order to avoid false optimism in efforts to increase physical activity to recommended levels.

The number of measured days might not represent the child's actual physical activity since this varies over time and between seasons (13). The extrapolated data from the diaries enabled three days of data collection in our study and this may not have been a sufficient period of time. There is no consensus about the optimal number of days of accelerometer use to estimate weekly physical activity behaviour, although it has been suggested that a period of three to seven days provides sufficient data (25).

There are various suggestions regarding the best placement for an accelerometer depending on the purpose of the investigation (26). In this study no difference was observed between the two sides, indicating that measurements on one side were sufficient (data not shown). A higher daily AEE was measured at the wrists than the ankles in both groups (data not shown) and daily AEE based on the wrist accelerometers was in accordance with the diaries. Possible explanations could be that accelerometers placed on the ankles tended to miss upper body activity during sedentary behavior and that the wrist placement also measured fidgeting (27). Comparing results of different placements are difficult and are considered inappropriate (27). Since the cut-off points differ between accelerometer brands and depend on individual variables, comparisons of study results are 
hampered (28). There are only limited published cut-off points for activity levels using the Actical at different places on children $(19,22)$.

Measurement of BMR by indirect calorimetry has been regarded as more valid than calculating BMR (29), although less invasive. This study showed good agreement between the $B M R_{m}$ and the BMR calculated by the Schofield equation, so the $\mathrm{BMR}_{\mathrm{m}}$ may not be necessary in all settings. As the DIT only represents a small proportion of TEE, and does not show great fluctuation over short time periods (4), this study assessed TEE minus DIT and not the total TEE to be more precise, but this difference should not affect the results.

The diary we used for self-reports of physical activity has been evaluated for adolescents and not for younger children, which might have affected the result. The reason for choosing this specific method was that there was no other diary at the time of the study that had been validated for assessing daily AEE in children. Considering that this study was mainly based on comparisons of intra-individual data as opposed to inter-individual data, we assumed that it was possible to go beyond the age that the diary had been evaluated for (21). Despite the inaccuracy of self-report instruments (17) a diary can still be used to increase the self-awareness of individual physical activity behaviour, which can also increase motivation.

A positive side effect that we experienced with the accelerometers was that physical activity could be visualised and these graphs were helpful in following up any discussions with the child and caregiver.

\section{CONCLUSION}

In this study, children with cerebral palsy were shown to have low daily activity energy expenditure. They did not achieve the recommended physical activity levels laid out in the Nordic Nutrition Recommendations, but then neither did all of the age and gender matched controls. Physical activity was overestimated in self-reports in the diaries when they were compared with ankle accelerometer measurements in both groups, but this was not the case for the wrist measurements. 


\begin{abstract}
Abbreviations
AEE, activity energy expenditure; BMI, body mass index; BMR, basal metabolic rate; DIT, diet-induced thermogenesis; NNR, Nordic nutrition recommendations; TEE, total energy expenditure.
\end{abstract}

\title{
ACKNOWLEDGEMENTS
}

The authors thank all the children and their families who took part in the study. The authors also thank Associated Professor Aili Low for extensive help with finalising the manuscript.

\section{FUNDING}

This study was funded by the Norrbacka Eugenia Foundation and the Royal Court Wedding Foundation. The funding agencies had no influence on the preparation of this paper.

\section{CONFLICT OF INTERESTS}

The authors have no conflict of interests to declare.

\section{References}

1. WHO. The World Health report 2002 - reducing risks, promoting healthy life. Geneva: World Health Organization 2002. http://www.who.int/whr/2002/en/

2. Kallings L. Physical activity on prescription: studies on physical activity level, adherence and cardiovascular risk factors. Thesis: Karolinska Institutet, Stockholm: 2008: 2-3.

3. Craggs C, Corder K, van Sluijs E, Griffin S. Determents of change in physical activity in children and adolescents. A systematic review. Am J Prev Med 2011; 40: 645-58.

4. Becker W, Lyhne A, Pedersen A, Aro M, Fogelholm I, Pho'rsdottir S et al. "Nordic Nutrition Recommendations: Integrating nutrition and physical activity." Scand J Clin Nutr 2004; 48: 178-87. 
5. American College of Sports Medicine. Guidelines for exercise testing and prescription $6^{\text {th }}$ ed. Lippincott: Baltimore: Williams \& Wilkins, 2000.

6. Schoefield W, Schofield C, James W. Basal metabolic rate: Review and prediction. Hum Nutr Clin Nutr 1985; 39: 1-96.

7. Maher C, Williams M, Olds T, Lane A. Physical and sedentary activity in adolescents with cerebral palsy. Dev Med Child Neurol 2007; 49: 450-7.

8. Fowler E, Kolobe T, Damiano D, Thorpe D, Morgan D, Brunstrom J et al. Promotion of physical fitness and prevention of secondary conditions for children with cerebral palsy: section on paediatrics research summit proceedings. Phys Ther 2007; 87: 1495-510.

9. Rosenbaum P, Paneth N, Leviton A, Goldstein M, Bax M. A report: the definition and classification of cerebral palsy. Dev Med Child Neurol 2006; 49: 8-14.

10. Beckung E, Hagberg G. Neuroimpairments, activity limitations and participation restrictions in children with cerebral palsy. Dev Med Child Neurol 2002; 44: 309-16.

11. Palisano R, Rosenbaum P, Walter S, Russell D, Wood E, Galuppi B. Development and reliability of a system to classify gross motor function in children with cerebral palsy. Dev Med Child Neurol 1997; 39: 214-23.

12. Capio C, Sit C, Abernethy B. Physical activity measurement using MTI (Actigraph) among children with cerebral palsy. Arch Phys Med Rehabil 2010; 91: 1283-90.

13. Dencker M, Andersen L. Health-related aspects of objectively measured daily physical activity in children. Clin Physiol Funct Imag 2008; 28: 133-44.

14. Verschuren O, Ketelaar M, Gorter J, Helders P, Uiterwaal C, Takken T. Exercise training program in children and adolescents with cerebral palsy: a randomized controlled trial. Arch Ped Adolesc 2007; 161:1075-81

15. L Bratteby Tollerz, R Olsson, A Forslund, S Norrlin. Reliability of energy cost calculations in children with cerebral palsy, cystic fibrosis and healthy controls. Acta Paediatr 2011; 100: 1616-20.

16. Mattsson E. Energy cost of level walking. Scand J Rehab Med 1989 Suppl 23: 1-48.

17. Adamo K, Prince S, Tricco A, Connor-Gorber S, Tremblay M. A comparison of 
indirect versus direct measures for assessing physical activity in the pediatric population: a systematic review. Int J Ped Obes 2009; 4: 2-27.

18. Eston R, Rowlands A, Ingledew D. Validity of heart rate, pedometry and accelerometry for predicting the energy cost of children`s activities. Appl Physiol 1998; 84: 362-71.

19. Rosenkranz, R, Rosenkranz S, Weber C. Validity of the Actical accelerometer step-count function in children. Ped Exerc Sci 2011; 23: 355-65.

20. Karlberg J. Body mass index reference values (mean and SD) for Swedish children. Acta Paediatr 2001; 90: 1427-34.

21. Bratteby LE, Sundhagen B, Fan H, Samuelson G. A 7-day activity diary for assessment of daily energy expenditure validated by the doubly labelled water method in adolescents. Eur J Clin Nutr 1997; 51: 585-91.

22. Heil D. Predicting activity energy expenditure using the Actical activity monitor. Res Qt Exerc Sport 2006; 77: 64-80.

23. Holm S. A simple sequentially rejective multiple test procedure. Scand J Statist 1979; 6: 65-70.

24. Shepherd R. Limits to the measurement of habitual physical activity by questionnaires. Br J Sports Med 2003; 37: 197-206.

25. Matthews C, Hagstromer M, Pober D, Bowles H. Best practises for using physical activity monitors in population-based research. Med Sci Sports Exerc 2012; 44: 68-76.

26. Guinhoya C, Hubert H, Dupont G, Durocher A. Relationship between the MTI Accelerometer (Actigraph) counts and running speed during continuous and intermittent exercise. Exerc J Sports Sci Med 2005; 4: 534-42.

27. Routen A, Upton D, Edwards M, Peters D. Discrepancies in accelerometer-measured physical activity in children due to cut-point non-equivalence and placement site. J Sports Sci 2012; 30: 1303-10.

28. Anderson C, Hagstromer M, Yngve A. Validation of the PDPAR as an adolescent diary: effect of accelerometer cut points. Med Sci Sports Exerc 2005; 37: 1224-30. 
29. Haugen H, Chan L-N, Li F. Indirect calorimetry: a practical guide for clinicians. Nutr Clin Pract 2007; 22: 377-88. 